\title{
Beneficio de los ejercicios de natación en la preparación y rendimiento de los futbolistas de 13-14 años
}

Benefit of swimming exercises in the preparation and performance of 13-14 year old players

Alex Perlaza Estupiñan ${ }^{* *}$

Flavio Arturo Perlaza Concha**

Manuel Gutiérrez Cruz ${ }^{* * *}$

\section{Resumen}

Las tendencias fisiológicas del entrenamiento deportivo determinan al fútbol como una actividad deportiva, está compuesta de una variedad de gestos repetitivos durante todo el desarrollo del partido Objetivo. Implementar un manual de ejercicios de natación que ayuden a mejorar el rendimiento físico de los futbolistas. Materiales y métodos. Investigación de tipo descriptiva Resultados. Se utilizó la encuesta para evaluar a los 10 entrenadores, el cálculo porcentual.

\footnotetext{
* Artículo original derivado del Proyecto "Beneficio de los deportes" Financiado por F.E.D.E.R y Instituto tecnológico superior de fútbol entre agosto 2017 y mayo 2019

** Docente universitario, F.E.D.E.R, Instituto tecnológico superior de fútbol. Guayaquil, Ecuador. E-mail: alex.perlaza@itsf.edu.ec. ORCID: http://orcid.org/0000-0001-8277-7806.

*** Docente universitario, Facultad de Educación Física deportes y recreación. Guayaquil, Ecuador. E-mail: flavio.perlazac@ug.edu.ec. ORCID: http://orcid.org/0000-0001-8036-4979.

**** Docente universitario, Facultad de Educación Física deportes y recreación. Guayaquil, Ecuador. E-mail: gutierrezcruz_manuel@yahoo.com. ORCID: http://orcid.org/0000-0001-72899623.
}

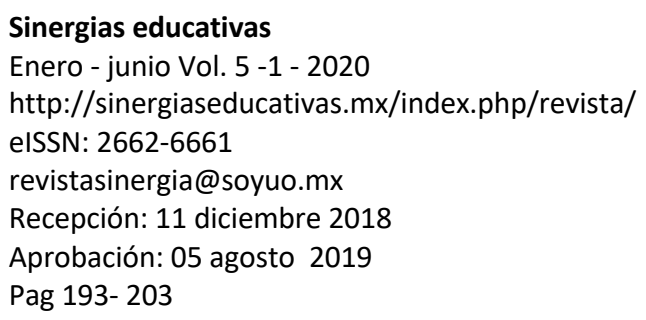

Disponible en

http://www.redalyc.org/articulo.oa?id=57356143200 14

Atribución/Reconocimiento-NoComercialCompartirlgual 4.0 Licencia Pública Internacional CC BY-NC-SA 4.0

https://creativecommons.org/licenses/by-ncsa/4.0/legalcode.es 
Conclusión. Proponer el manual de ejercicios de natación para mejorar el rendimiento físico.

Palabras clave: rendimiento físico, fuerza, velocidad, resistencia, natación.

\begin{abstract}
The physiological tendencies of sports training determine football as a sporting activity, it is composed of a variety of repetitive gestures throughout the development of the match. Objective. Implement a manual of swimming exercises that help improve the physical performance of soccer players. Materials and methods. Descriptive type research Results. The survey was used to evaluate the 10 coaches, the percentage calculation. Conclusion. Propose the manual of swimming exercises to improve physical performance.
\end{abstract}

Key words: physical performance, strength, speed, endurance, swimming.

\title{
Introducción
}

El deporte del fútbol hoy en día es uno de los fenómenos sociales más relevantes de nuestra cultura, siendo la forma más común de entender la actividad física en la sociedad ecuatoriana. Sin embargo, la práctica óptima de este deporte a nivel profesional requiere un alto nivel de rendimiento físico. El fútbol como práctica deportiva en equipo, es una de las actividades preferidas de los adolescentes.

Mientras más alto sea el desarrollo del componente físico en todos los futbolistas de un equipo, mayores serán las posibilidades de alcanzar el éxito, pues a pesar de tratarse de un deporte colectivo, constantemente suceden acciones individuales que en ocasiones deciden sobre el resultado del juego. Además en el caso del fútbol a pesar de que se juega más sin balón que con balón, no se trata de correr por correr, sino de hacerlo con una idea para jugar mejor.

La actividad física que desarrollan los niños y adolescentes crea cambios en su organismo que trae como consecuencia adaptaciones fisiológicas en el organismo dentro de las condiciones físicas que 
sufren mayor cambio durante el crecimiento tenemos a la fuerza, flexibilidad, velocidad y resistencia.

Para los niños y adolescentes el ejercicio físico tiene como objetivo principal ayudar a mejorar los procesos de crecimiento y desarrollo favoreciendo un estímulo para el sistema metabólico, siempre y cuando este bien organizado y planificado.

La natación ayuda a mejorar la capacidad respiratoria o el VO2 máximo, que es la capacidad máxima de oxígeno que el organismo puede absorber, transportar y consumir en un tiempo determinado, es decir, el máximo volumen de oxígeno en la sangre que nuestro organismo puede transportar y metabolizar, además, mejora la capacidad física aeróbica y anaeróbica. Todos estos son aspectos fundamentales para el desarrollo físico del futbolista.

La natación es especialmente eficaz cuando se trata de promover mejoras en el sistema cardiovascular. De hecho, la natación regularmente puede mejorar la contractilidad del corazón, disminuir la presión arterial, disminuir la frecuencia cardíaca y mejorar la eficiencia de los pulmones. (Mieles, 2017, p24).

Actualmente a los jóvenes se los involucra en una actividad deportiva desde temprano edad. Para realizar esta actividad cada entrenador tiene la obligación de conocer cuales con las particularidades anatómicas que posee cada niño y adolescente para la práctica de los ejercicios físicos, cabe recalcar que los ejercicios físicos que se impongan no deben afectar el desarrollo óseo de los niños y adolescentes.

A la edad de 13 a 14 años es donde los hombres pueden aumentar la fuerza ya que presentan cambios de testosterona adecuados para su fortalecimiento, en cambio en las mujeres este cambio se presenta a la edad de 11 y 12 años ya que a esta edad es cuando se presenta la menarquia la velocidad es un factor que se presenta a las 10 años y ya a las 14 años de edad se mejora debido a la fuerza explosiva.

Lucero et al, (2015), manifiesta que la natación ofrece a los niños y jóvenes beneficios como: 
Permite que desarrollen su multilateralidad, un adecuado desarrollo físico y psicológico.

Favorece al progreso en las cualidades físicas básicas como la resistencia, fuerza, velocidad y flexibilidad.

Ayuda al desarrollo de la coordinación motriz y el equilibrio.

Contribuye al desarrollo de los huesos y músculos.

Aumenta la seguridad y auto estima.

Ayuda a la correcta alineación vertebral por tanto a la reducción postural. (p22)

La natación es un deporte que puede ayudar notoriamente a los jugadores de fútbol para mantenerse en forma durante la temporada baja y puede servir como un entrenamiento cruzado para reducir el riesgo en las lesiones, los entrenamientos de natación también ayudan a construir una resistencia cardiovascular en los deportistas, sin embargo la mecánica de una patada agitada puede ser un desafío para los jugadores.

En esta investigación nuestro objetivo principal fue poder desarrollar un manual de ejercicios acuáticos para identificar los beneficios que causaría la natación en el rendimiento de un futbolista para la cual realizamos una investigación tomando fundamentos teóricos de diferentes fuentes bibliográficas para poder averiguar cuáles son las falencias que ocurren en el rendimiento deportivo de un futbolista.

Los adolescentes de 13-14 años pertenecientes a las ligas menores del Club Real Fortaleza se encuentran cursando la educación secundaria, por tanto, no pueden dedicarse a tiempo completo al entrenamiento físico de alto rendimiento que necesita un futbolista para desarrollar todo su potencial. Debido a estas razones no reciben un entrenamiento sistemático especial de acuerdo a sus condiciones físicas, fisiológicas, psicológicas y de tiempo para lograr el desarrollo de su rendimiento físico.

- Escasa preocupación de los entrenadores. 
- Mala preparación física y deportiva en las etapas iniciales.

- Poca disposición de tiempo de los adolescentes.

Consecuencias

- Fracaso de aspiraciones de convertirse en jugador profesional.

- Baja autoestima y motivación por su entorno familiar en el deportista.

- Escasez de deportistas profesionales bien preparados.

De esta situación problemática se deriva el problema científico al cuestionarse que, ¿De qué manera beneficiaria la influencia de ejercicios de natación el rendimiento delos futbolistas de 13-14 años del Club Real Fortaleza en Guayaquil?

Este trabajo es factible, debido a que se desarrollará en las instalaciones del Club de fútbol Real Fortaleza, provista de la infraestructura y los entrenadores necesarios para la fase metodológica. Este se constituye en un proyecto que aportaría a la formación de decenas de nuevas promesas del deporte que se beneficiarían con la propuesta de un manual de ejercicios posturales mediante la natación para para el mejoramiento del rendimiento físico de los futbolistas juveniles.

\section{Actividad Física y Deportiva en Niños y Adolescentes}

Actualmente a los niños se los involucra en una actividad deportiva desde temprano edad. Para realizar esta actividad cada entrenador tiene la obligación de conocer cuales con las particularidades anatómicas que posee cada niño y adolescente para la práctica de los ejercicios físicos, cabe recalcar que los ejercicios físicos que se impongan no deben afectar el desarrollo óseo de los niños y adolescentes.

Dentro de los factores que afectan el crecimiento en el desarrollo de los niños y adolescente tenemos a los intrínsecos, que son los que se encuentran en la herencia ya que los niños tiene una fuerte 
correlación con la estatura del padre, el sexo es muy importante ya que hasta los 10 años la diferencia entre los hombres y mujeres es muy poca pero a partir de la pubertad alcanzan con anterioridad la estatura definitiva, en los factores extrínsecos tenemos a la nutrición esta es de vital importancia ya que una alimentación balanceada y equilibrada es pieza clave para el potencial genético del niño .

La actividad física que desarrollan los niños y adolescentes crea cambios en su organismo que trae como consecuencia adaptaciones fisiológicas en el organismo dentro de las condiciones físicas que sufren mayor cambio durante el crecimiento tenemos a la fuerza, flexibilidad, velocidad y resistencia.

A la edad de 13 a 14 años es donde los hombres pueden aumentar la fuerza ya que presentan cambios de testosterona adecuados para su fortalecimiento, en cambio en las mujeres este cambio se presenta a la edad de 11 y 12 años ya que a esta edad es cuando se presenta la menarquia la velocidad es un factor que se presenta a las 10 años y ya a las 14 años de edad se mejora debido a la fuerza explosiva.

Cuando se tiene un mal uso de los implementos deportivos es una de las causas más comunes para las lesiones de los futbolistas ya que un material dañado o con defectos va a causar técnicas defectuosas y sobrecargas para que no ocurran este tipo de errores se deben adecuar los implementos a la edad, peso, estatura de los deportistas.

El calentamiento es de vital importancia ya que ayuda y evita las lesiones además también a la preparación psicofísica y coordinativa del futbolista por lo cual es muy necesaria antes del entrenamiento, para poder aplicar de manera adecuada las cargas de entrenamiento existen diversos aspectos que deben ser tomados en cuenta tales como:

- Las niñas maduran fisiológicamente entre 2 y 2.5 años antes que los niños.

- La dieta adecuada es muy importantes cuando se realizan actividades físicas. 
- La anchura la densidad y la fuerza ósea son las principales afectaciones que sufre el organismo en un entrenamiento.

- El crecimiento en estatura es muy rápido en los 2 primeros años de vida, durante los cuales los niños alcanzan el $50 \%$ de la estatura de los adultos a los años de edad. Después de esto el ritmo es más lento a lo largo de la infancia hasta que se produce un notable incremento poco antes de la pubertad.

- Las lesiones en la epífisis pueden ocasionar la finalización prematura del crecimiento.

- La masa muscular aumenta de forma sostenida junto con el incremento de peso desde el nacimiento y durante la adolescencia.

- La intensidad de la acumulación de grasa depende de los hábitos dietéticos, ejercicio y de la genética.

- El equilibrio, la agilidad, y la coordinación mejoran a medida que el SNC en los niños se va desarrollando.

- La habilidad motora aumenta a lo largo de los primeros 18 años, en las niñas se estabiliza en la pubertad, esto se debe al aumento de estrógenos y a la vida sedentaria que llevan.

- La fuerza mejora cuando la masa muscular aumenta.

- El VO2 Max expresado en 1/min llega a su punto más alto entre los 17 y 21 años en los niños y entre $\operatorname{los} 12$ y 15 años en las niñas, después solo se logra mantenerlo.

- Los niños no pueden tener elevados intercambios respiratorios durante la realización de ejercicios máximos esto debido a una limitación en la producción de lactato. Por tanto la producción de potencia anaeróbica es menor.

- Las El riesgo de lesiones en deportistas jóvenes por entrenamiento contra resistencia es nulo y el programa a seguir es similar al del adulto. 
- El entrenamiento Aeróbico en adolescentes no mejora el VO2 Max, esto porque depende más del tamaño del corazón que del entrenamiento, pero la capacidad de resistencia si mejora.

- La capacidad Anaeróbica de un niño aumenta con el entrenamiento anaeróbico.

- Reducir la grasa corporal

- Incrementar la masa magra

- Incrementar la masa corporal total

\section{Materiales y métodos}

Esta investigación es de tipo descriptiva, llamadas también investigaciones diagnósticas. La misma se realizó en el Club Real Fortaleza de la ciudad de Guayaquil. La población estuvo conformada por los 10 entrenadores y 80 jóvenes seleccionando una muestra intencional de 30 jugadores de sexo masculino de 13-14 años de edad, siendo este el principal criterio de inclusión. Dentro de los métodos investigativos empleados se encuentran métodos teóricos tales como: (Histórico- Lógico, Análisis - Síntesis, Inductivo -Deductivo y el Sistémico Estructura Funcional, el cual permitió elaborar la propuesta). Dentro de los Métodos Empíricos, se empleó la observación, y cómo técnicas se utilizaron la encuesta para evaluar a los 10 entrenadores y el cálculo porcentual.

\section{Resultados}

Análisis de los resultados de las preguntas de la encuesta sobre la interrogante planteada se considera que el $90 \%$ de los entrenadores está de acuerdo en que los ejercicios anaeróbicos ayudan a tener mayor resistencia física muscular ya que el organismo humano tiene la capacidad biológica para adaptarse a exigencias de esfuerzo cada vez mayores

El 70\% considera que es importante una adecuada selección de ejercicios a la hora de diseñar un programa de ejercicios acuáticos de acondicionamiento neuromuscular, en este sentido al adecuación 
del ejercicio repercute previamente sobre la seguridad eficacia y funcionalidad del mismo.

El resultado de la encuesta indica que el $85 \%$ dijo que se obtendrían buenos resultados ya que mejorara la resistencia, flexibilidad, y fuerza y también podrá evitar varias lesiones.

EL $75 \%$ de los entrenadores considera que se obtendrán buenos resultados a partir del 4 o 5 entrenamientos viendo mejoras significativas en su cuerpo y en su habilidad EL $90 \%$ de los entrenadores indico que mediante la implementación de estos ejercicios mejorara la resistencia combinándolos con ejercicios de cardio en la piscina ayudando a fortalecer sus actividades en el entrenamiento.

Consecuentemente podemos deducir que el $80 \%$ de los entrenadores dijo que es muy beneficioso para la preparación en general ya que otorga beneficios físicos, psíquicos y fisiológicos que envolverán el desarrollo orgánico en los aspectos musculares y la coordinación motriz.

El 65\% de los entrenadores indicó que no conoce los efectos de la práctica de ejercicios acuáticos, pero están de acuerdo que deberían realizarse ya que mejora o mantiene los niveles de movilidad muscular proporcionando una tonificación general adecuada $\mathrm{y}$ evitando sobrecargas de trabajo en zonas concretas.

Las estadísticas indican que un gran porcentaje piensa que estos ejercicios van a mejorar la construcción muscular y movilidad aumentando la reserva energética de cada futbolista.

El 70\% de los entrenadores indica que los futbolistas mejoraran sus condiciones físicas y podrán aumentar su velocidad, flexibilidad, fuerza y resistencia en los entrenamiento deportivos obtienen mejoras notables en los partidos.

El 100\% concluyo que son esenciales para los futbolistas por que alcanzaran mediante estos ejercicios acuáticos un estado físico óptimo que les permitirá desarrollar un buen entrenamiento y una buena competencia en los partidos futuros. 
El desarrollo de ejercicios de natación que beneficie la formación física de los jóvenes futbolista, permitirá a los entrenadores ampliar los métodos de entrenamiento para fortalecer los estímulos multilaterales a los que los deportistas en formación, necesitan ser sometidos para que tengan el crecimiento adecuado tanto de las capacidades condicionales (fuerza, resistencia, velocidad, flexibilidad), como las coordinativas.

\section{Discusión}

En este proyecto nuestro objetivo principal fue poder identificar cuáles eran los beneficios que causaría la natación en el rendimiento de un futbolista para la cual realizamos una investigación tomando fundamentos teóricos de diferentes fuentes bibliográficas para poder averiguar cuáles son las falencias que ocurren en el rendimiento deportivo de un futbolista.

En la investigación se realizó una encuesta la cual nos iba a indicar cuáles son las falencias en este caso de los deportistas de 13-14 años del club de futbol Real Fortaleza de Guayaquil para poder identificar cuáles son los ejercicios de natación más adecuados para la situación de rendimiento en la que se encuentran actualmente los futbolistas.

\section{Conclusiones}

Luego de haber obtenido los resultados de dicha encuesta pudimos lograr saber con exactitud cuáles eran los ejercicios más adecuados de natación para poder mejorar el rendimiento de los futbolistas y así poder aumentar su nivel de competencia en los partidos de futbol.

Realizar capacitaciones a los entrenadores de fútbol para que aumenten sus conocimientos en su área y puedan así poder desarrollar nuevas formas que sean beneficiosas para aumentar el rendimiento de los futbolistas de 13-14 años en el club.

Realizar un estudio a profundidad de cuáles serían todos los beneficios de la natación que influyen en el rendimiento de los futbolistas tales como la fuerza, la flexibilidad, la velocidad, la coordinación y la resistencia y poder mejorar el desempeño deportivo en los niños de 13-14 años. Análisis sobre las ventajas de 
la natación a los futbolistas en cuanto a la recuperación de sus lesiones utilizando ejercicios en la piscina para poder lograr una recuperación más rápida y a la vez ayudar en la relajación muscular y lograr mejorar su nivel competitivo y rendimiento en sus entrenamiento.

\section{Referencias}

José Manuel Morejón Rodríguez, Osvaldo Javier Martín Agüero, Jesús Font Landa., y René Pérez Mestre. (2015). La condición física en el fútbol. ¿Para correr más o para jugar mejor? Ventajas de los métodos intermitente, simulado e integrado en la preparación del futbolista. Efdeportes. Lectura de educación fisica $y$ deportes, 20(205). Obtenido de https://www.efdeportes.com/efd205/la-condicion-fisica-en-el-futbol.htm

Lucero Sarmiento Monica Gabriela, Maza Camas Manuel Pedro. (2015). Metodología para el aprendizaje de la natación en los tiños de tercero y cuarto de básica en la Unidad Educativa Asián Américan Scholl. Cuenca (tesis de grado). universidad politécnica salesiana. Cuenca, Ecuador

Mieles Moreira, O. J. (2016). Beneficio de los ejercicios de natación en la preparacion y rendimiento de los fútbolistas de 13-14 años.(Tesis de grado) Universidad de Guayaquil: Guayaquil, Ecuador.

News., S. (2014). Sandranews.com. Obtenido de http://www.sandranews.com/natación-par-el-futbol/

Tobón, E. R. (2011). Actividad Física y Deportiva en Niños y Adolescentes. Educación física salud y recreación. Recuperado de: $\mathrm{http} / / /$ rolerioredufisica.blogspot.com/p/educacion-fisica-910-y11.html

Weineck, J. E. (1996). Fútbol total. el entrenamiento fisico del futbolista. Barcelona: Paidotrobo. 